\title{
Shared Presence in Physician-Patient Communication: A Graphic Representation
}

\author{
William B. Ventres \\ Institute for Studies in History, Anthropology, and Archeology, University of El Salvador, and \\ Department of Family Medicine, Oregon Health \& Science University;
}

Richard M. Frankel

Regenstrief Institute, IU/Simon Cancer Center, Indiana University School of Medicine

Acknowledgement: The authors thank Frederick Barken, MD, Ronald Epstein, MD, and Thomas Inui, MD, for their thoughtful comments on this work. They also thank Vi Luong for designing the graphics.

\begin{abstract}
Shared presence is a state of being in which physicians and patients enter into a deep sense of trust, respect, and knowing that facilitates healing. Communication between physicians and patients (and, in fact, all providers and recipients of health care) is the medium through which shared presence occurs, regardless of the presenting problem, time available, location of care, or clinical history of the patient. Conceptualizing how communication leads to shared presence has been a challenging task, however. Pathways of this process have been routinely lumped together as the biopsychosocial model or patient, person, and relationship-centered care--all deceptive in their simplicity but, in fact, highly complex--or reduced to descriptive explications of one constituent element (e.g., empathy). In this article, we reconcile these pathways and elements by presenting a graphic image for clinicians and teachers in medical education. This conceptual image serves as a framework to synthesize the vast literature on physician-
\end{abstract}

This is the author's manuscript of the article published in final edited form as:

Ventres, W. B., \& Frankel, R. M. (2015). Shared presence in physician-patient communication: A graphic representation. Families, Systems, \& Health, 33(3), 270-279. http://doi.org/10.1037/fsh0000123 
patient communication. We place shared presence, the fundamental characteristic of effective clinical communication, at the center of our figure. Around this focal point, we locate four elemental factors that either contribute to or result from shared presence, including interpersonal skills, relational contexts, actions in clinical encounters, and healing outcomes. By visually presenting various known and emergent theories of physician-patient communication, outlining the flow of successful encounters between physicians and patients, and noting how such encounters can improve outcomes, physicians, other health care professionals, and medical educators can better grasp the complexity, richness, and potential for achieving shared presence with their patients.

Keywords: communication barriers, medical philosophy, outcome assessment (health care), physicianpatient relations, physician's role 
A mysterious kind of feeling [occurs] when you and a patient are in a room together, and you're either talking about something or sharing an important moment. You can just tell. You feel extraordinarily connected to them, and they feel extraordinarily connected to you in some important way. The feeling that they have is . . . therapeutic to them . . . and it's incredibly rewarding to you.-Physician interview (Frankel, Inui, Karnieli-Miller, \& Taylor, 2010, p. 5)

Physicians know when this feeling happens. Patients do, too. So do their companions and family members. The experience is one of shared presence. Shared presence happens when physicians, patients, and others come together in clinical settings; when they fully engage in the timeless healing rituals of diagnosis, treatment, teaching, and learning; and when they part knowing that a deeply felt therapeutic process has occurred. Shared presence is thus an interpersonal phenomenon that involves knowing, as well as knowing that the other knows, that time together is being shared in a deeply meaningful way. Although they arise against a background of routine practice, moments of shared presence are special. Communication between physicians and patients (or, in fact, between any providers and recipients of health care) is the medium through which shared presence occurs, regardless of presenting concern, location of care, time available, or clinical history of the patient (Suchman, Markakis, Beckman, \& Frankel, 1997). Communication, in turn, is based on an intricate network of social, psychological, structural, and biomedical elements that occur in the common milieu of time and space (Epstein et al., 2005). Conceptualizing how communication leads to shared presence has been a challenging task. Pathways of this process have been routinely lumped together as the biopsychosocial model or patient, person, and relationship-centered care—all deceptive in their simplicity but, in fact, highly complex—or reduced to descriptive explications of one constituent element (e.g., empathy), thereby becoming narrowly focused and lacking integration with practice realities (Frankel, Quill, \& McDaniel, 2003; Levinson, Lesser, \& Epstein, 2010; Starfield, 2011; Suchman, 2006).

Our purpose here is to reconcile these pathways and elements into a graphic image that can serve, especially for clinicians and teachers in medical education, as a framework for synthesizing the vast 
literature on physician-patient communication (Figure 1 and Table 1). We place shared presence at the center of Figure 1. Around this focal point, we locate four sets of elemental factors that either contribute to or result from shared presence, including interpersonal skills, relational contexts, actions in clinical encounters, and healing outcomes. In subsequent figures and associated text, we present a holistic summary of these sets and factors. By visually presenting well-known and emerging theories of physician-patient communication, outlining the flow of successful encounters between physicians and patients (Charon, 2001; Epstein \& Street, 2011; Frankel \& Stein, 2001; Wilson, 2000), and noting how such encounters can improve clinical outcomes (Street, Makoul, Arora, \& Epstein, 2009), we believe physicians and educators can better grasp the complexity, richness, and potential for achieving shared presence with their patients.

\section{Review of Shared Presence}

Although many physicians may be unaware of how they engage in communication with patients or how their patterns of interaction may influence clinical conditions, all have at one time or another experienced a sense of intense emotional connectedness with one or more of those in their care. This connectedness — shared presence — is a state of being in which physicians and other health professionals and their patients enter into a deep sense of trust, respect, and knowing that facilitates healing. It is a communicative state during which both physicians and patients feel a richly personalized, intimate, and profoundly meaningful therapeutic event is occurring. Shared presence is as much felt as considered, a “bonded resonance” between individuals mediated by qualities such as authenticity and intimacy. It is fostered by the ability to recognize fully the existence of self, other, and all that lies in between (McPhee, 1981). When presence is shared, patients feel known, understood, and able to cope with both disease and illness; physicians sense the existence of a connection that is therapeutic independent of the biomedical treatments they offer (Matthews, Suchman, \& Branch, 1993; Suchman \& Matthews, 1988). 


\section{Interpersonal Skills}

To cultivate shared presence, physicians first develop awareness and inquisitiveness as specific interpersonal skills for exploring patients' concerns (Figure 2). These skills are based on physicians' abilities to practice self-reflection, accept differences in perspectives, consider alternate expectations, and adjust their interactive style accordingly. Fundamental to developing these skills is acknowledging that two or more points of view exist in every encounter with patients (Eisenberg, 1977; Ventres \& Haq, 2014), a circumstance influenced by multiple factors such as families of origin (Mengel \& Mauksch, 1989), power and its expression in clinical dialogues (Beisecker, 1990), and one’s capacity to remain mindful or reflective in the moment (Epstein, 1999). For physicians, a number of communicative approaches exist, and no one strategy is appropriate in all situations (Helman, 2007) Practically speaking, developing interpersonal skills means working to "see” patients as persons. This means recognizing and inquiring about patients’ uniquely human responses to suffering, not just asking about presenting problems. "Seeing” patients is accomplished by

- Demonstrating genuine concern through the expression of curiosity (Jagosh et al., 2011)

- Listening attentively to patients’ presentations and noting special concerns underlying patients’ narratives (Mishler, Clark, Ingelfinger, \& Simon, 1989)

- Conducting the physical examination thoughtfully, consciously applying touch and observing patients’ reactions (Verghese, 2009; Verghese, Brady, Kapur, \& Horwitz, 2011)

- Observing with sensitivity ambient feelings, both those expressed by patients as well as those experienced by oneself (Halpern, 2003)

- Considering carefully the use of words and time in the creation of appropriate therapeutic agendas (Coulehan et al., 2001; Mauksch, Dugdale, Dodson, \& Epstein, 2008) 


\section{Relational Contexts}

Second, physicians invite shared presence by integrating psychological, social, and spiritual contexts of care into their clinical encounters with patients (Figure 3). Some contextual factors focus on relationships. Family members, companions, caregivers, and medical consultants at one time or another all play significant roles in patient care. Some are organizational—for example, knowing what economic and professional resources are available and how they can be utilized (Ventres \& Frankel, 2010) or whether patients are literate in regards to health information (Weiss, 2014). Some contextual factors are cultural in origin, such as personal religious beliefs and social determinants of health (including racism, sexism, and poverty, among others; Schouten \& Meeuwesen, 2006).

Understanding these multiple contexts is one step toward optimizing the potential for shared presence. Other steps include exploring the effects of context on functioning in daily life and acknowledging their influence on disease. Integrating these insights in clinical interactions involves seeing beyond patients’ limitations toward appreciating the strengths they exhibit in the midst of illness experiences (Ventres, 2012a).

\section{Actions in the Clinical Encounter}

Third, physicians nurture shared presence through their direct actions with patients, recognizing that every clinical encounter takes place in the present, has a beginning and an end, is linked to the past with implications for the future, and has identifiable activities that occur simultaneously as well as sequentially (Frankel \& Stein, 2001; Figure 4). These identifiable activities or habits of practice include building connections with patients, eliciting their perspectives, demonstrating empathy for their concerns, and educating patients with intent to motivate healthy behaviors. Each is accomplished in and through the use of language and behavior. Each facilitates the task of thoughtfully and accurately identifying patients' symptoms and concerns (Feldman \& Berkowitz, 2012; Frankel \& Stein, 2001). Each is based on 
relational foundations that help determine outcomes. The intent of these actions is to seamlessly integrate compassionate concern and empathic understanding with medical history taking — the identification and reorganization of clinical concerns-knowing that each one informs the other and enhances the progression from presentation to accurate diagnosis and successful treatment (Finset, 2012).

\section{Healing Outcomes}

Fourth, out of shared presence emerge outcomes that contribute to healing, specifically process and therapeutic outcomes that reflect patients’ progressions toward wellness. Process outcomes are mutual emotional sentiments, experienced by both patients and physicians. They include intimacy, partnership, understanding, and trust (Carmichael \& Carmichael, 1981; Saba et al., 2006; Figure 5). Each of these emotional reactions results from ongoing attention to the dynamic interpersonal give-and-take that takes place in clinical encounters, as outlined in the sections above.

From these process outcomes, better therapeutic outcomes are achieved. These are improvements in quantifiable health-related conduct (notably increased adherence to treatment plans), clinical efficiency, and medical results (including positive changes in clinical conditions) that have been repeatedly demonstrated across various disciplinary boundaries (Mauksch et al., 2008; Rao, Anderson, Inui, \& Frankel, 2007). In the long term, shared presence can lead to empowering patients’ health-seeking behaviors, expanding their communicative capacities, and enhancing their social capital (Stewart et al., 2000).

\section{Discussion}

Many within medicine and other health professions have worked to describe the state of being we term shared presence, without using these specific words (Carmichael \& Carmichael, 1981; Epstein \& Street, 2011; Matthews et al., 1993; McPhee, 1981; Suchman \& Matthews, 1988). Shared presence has 
found some renown on canvas and as a short aphorism (Moore, 2008; Peabody, 1927), and there exist a variety of anecdotes from practice that patients and physicians have used to create images of its existence or absence. A Fortunate Man (Berger, 1997) offers excellent examples on both accounts.

Regardless of what one names it, whether shared presence or another term, our intent here has been to offer a graphic representation of this state of being. We have tried to visually frame those factors that work toward developing shared presence, among them creating welcoming healing environments, focusing on patients as people first, showing respect, fostering collaboration, cultivating participation, exploring resiliencies, and sharing realistic expectations at the same time imagining hopefulness. We have also tried to illustrate how shared presence improves relational and clinical outcomes of physician-patient encounters (Jagosh et al., 2011; Street et al., 2009), knowing full well that shared presence is a fluid, synthetic, and organic reality not controlled solely by either end of the relationship, the physician's or the patient's: it is a jointly created interactional achievement. We acknowledge, as well, that other graphic summaries might look different than ours.

Nonetheless, virtually all physicians can recall episodes during which they have shared presence with their patients. Given its universal character, shared presence is a deeply rooted goal of effective clinical communication beyond mere information transfer. Shared presence is of fundamental importance in generalist practice, with its emphasis on relationships built over time (Barken, 2011; Ventres, 2012a). Many scholars have also written about the value of inviting shared presence in moments of critical importance, including end-of-life discussions (Hallenbeck, 2005; Tulsky, 2005), when discussing medical error (Hébert, Levin, \& Robertson, 2001), assessing diagnostic and therapeutic uncertainty (Charles, Whelan, \& Gafni, 1999), and giving bad news (Rabow \& McPhee, 1999). We submit that some small aspect of shared presence can be found in every clinical conversation that purports to be therapeutic.

Many believe the utility of shared presence is rapidly disappearing in the face of new technologies, the changing focus of biomedical thinking and purpose, reimbursement strategies that favor procedures over time spent with patients, and a medical culture that has become increasingly market oriented over the past 50 years (Barken, 2011). Many more are beleaguered by the competing priorities of 
modern medical encounters, including demands for attention and time made by electronic medical records in examination rooms and hospital suites (Ventres \& Frankel, 2010). Although the nascent tools of modern medical practice and the digital revolution hold high potential for making critical information readily available to physicians, paradoxically they may also erode the core of human relationships needed to enhance healing. Without shared presence, patients feel ignored or even insulted, both of which have been linked to patient dissatisfaction and an increased risk of medical malpractice in the event of untoward clinical results (Levinson, Roter, Mullooly, Dull, \& Frankel, 1997).

Rather than simply dismissing all these forces as evil influences on the practice of medicine, however, we need to find ways to reinvigorate the emotionally rich therapeutic alliance of shared presence. Much of the communicative work of medicine can be shared with other professionals, including community health workers, nurses, behavioral scientists, and other clinical team members. Shared presence, on the other hand, cannot simply be delegated to others; for however trivial physicians may view presenting concerns, patients see them as vital: frustrations to be endured, illnesses to be managed, or true emergencies to be overcome. By attending to the factors in our graphic, we believe physicians can realize the depth of meaning that shared presence can bring as a teachable, learnable, and practicable way of being.

Being open to or facilitating shared presence is not just being empathetic or nice: a good person doing a good job in a caring and friendly manner. It is the result of working to recognize the shared humanity of every person who seeks a physician’s council and to convey a caring response in turn. Experienced physicians understand that the same high degree of shared presence is neither possible nor desired for every encounter (Miller, 1992); good physicians are open to its occurrence by creatively reaching into their professional repertoires and using the elements we review in daily practice. In doing so, they invite patients and their family members to respond in kind, creating a space for meaning to arise from mutual attention (Chance, 1976). Although meeting in this space may invoke distress on both sides of the stethoscope-abiding conflict and loss in the face of patients' illness experiences is not an uncommon experience for physicians (Makowski \& Epstein, 2012)—it can be cause for joy from which 
deep satisfaction may arise (Ventres, 2012b). For in addition to its communicative and clinical functions, shared presence also has a moral function: to bring alive the spirit of doctoring, placing the physicianpatient relationship at the center of the healing work that is the core of medicine.

\section{Conclusion}

Shared presence reflects a fundamental human desire for connection. In this article, we have linked this desire with a systematically organized graphic representation of its application in clinical settings. Although shared presence may not always be realized in physician-patient encounters, enhancing its potential is the result of known skills, practiced habits, and a keen awareness of both self-inrelationship with others and the physical, clinical, and emotional contexts in which these encounters take place. By reflecting on those times when such a connection has been experienced with patients, physicians and other health professionals can begin to explore the theoretical aspects, day-to-day dynamics, and beneficial therapeutic effects of shared presence in medical practice. 


\section{References}

Barken, F. M. (2011). Out of practice: Fighting for primary care medicine in America. Ithaca, NY: Cornell University Press.

Beisecker, A. E. (1990). Patient power in doctor-patient communication: What do we know?Health Communication, 2, 105-122. 10.1207/s15327027hc0202_4

Berger, J. (1997). A fortunate man. New York, NY: Random House USA.

Carmichael, L. P., \& Carmichael, J. S. (1981). The relational model in family practice. Marriage \& Family Review, 44, 123-133. 10.1300/J002v04n01_07

Chance, M. (1976). The social structure of attention. New York, NY: John Wiley.

Charles, C., Whelan, T., \& Gafni, A. (1999). What do we mean by partnership in making decisions about treatment?British Medical Journal, 319, 780-782. 10.1136/bmj.319.7212.780

Charon, R. (2001). The patient-physician relationship. Narrative medicine: A model for empathy, reflection, profession, and trust. JAMA: Journal of the American Medical Association, 286, 1897-1902. 10.1001/jama.286.15.1897

Coulehan, J. L., Platt, F. W., Egener, B., Frankel, R., Lin, C. T., Lown, B., \& Salazar, W. H. (2001). “Let me see if I have this right . ..”: Words that help build empathy. Annals of Internal Medicine, 135, 221227. 10.7326/0003-4819-135-3-200108070-00022 
Eisenberg, L. (1977). Disease and illness. Distinctions between professional and popular ideas of sickness. Culture, Medicine and Psychiatry, 1, 9-23. 10.1007/BF00114808

Epstein, R. M. (1999). Mindful practice. JAMA: Journal of the American Medical Association, 282, 833839. 10.1001/jama.282.9.833

Epstein, R. M., Franks, P., Fiscella, K., Shields, C. G., Meldrum, S. C., Kravitz, R. L., \& Duberstein, P. R. (2005). Measuring patient-centered communication in patient-physician consultations: Theoretical and practical issues. Social Science \& Medicine, 61, 1516-1528. 10.1016/j.socscimed.2005.02.001

Epstein, R. M., \& Street, R. L., Jr. (2011). Shared mind: Communication, decision making, and autonomy in serious illness. Annals of Family Medicine, 9, 454-461. 10.1370/afm.1301

Feldman, M. D., \& Berkowitz, S. A. (2012). Role of behavioral medicine in primary care. Current Opinion in Psychiatry, 25, 121-127. 10.1097/YCO.0b013e3283503576

Finset, A. (2012). “I am worried, doctor!” Emotions in the doctor-patient relationship. Patient Education and Counseling, 88, 359-363. 10.1016/j.pec.2012.06.022

Frankel, R. M., Inui, T. S., Ivy, S., Karnieli-Miller, O., \& Taylor, A. (2010). What happens when things go right? Using work life narratives to understand values in a large healthcare organization (Unpublished final report). Indianapolis, IN: Clarian Health Systems.

Frankel, R. M., Quill, T. E., \& McDaniel, S. H. (Eds.). (2003). The biopsychosocial approach: Past, present, future. Rochester, NY: University of Rochester Press. 
Frankel, R. M., \& Stein, T. (2001). Getting the most out of the clinical encounter: The four habits model. The Journal of Medical Practice Management: MPM, 16, 184-191.

Hallenbeck, J. (2005). Palliative care in the final days of life: “They were expecting it at any time.”JAMA: Journal of the American Medical Association, 293, 2265-2271. 10.1001/jama.293.18.2265

Halpern, J. (2003). What is clinical empathy?Journal of General Internal Medicine, 18, 670-674. 10.1046/j.1525-1497.2003.21017.x

Hébert, P. C., Levin, A. V., \& Robertson, G. (2001). Bioethics for clinicians: 23. Disclosure of medical error. Canadian Medical Association Journal, 164, 509-513.

Helman, C. G. (2007). Culture, health and illness (5th ed.). London, UK: Hodder Arnold.

Jagosh, J., Donald Boudreau, J., Steinert, Y., Macdonald, M. E., \& Ingram, L. (2011). The importance of physician listening from the patients’ perspective: Enhancing diagnosis, healing, and the doctor-patient relationship. Patient Education and Counseling, 85, 369-374. 10.1016/j.pec.2011.01.028

Levinson, W., Lesser, C. S., \& Epstein, R. M. (2010). Developing physician communication skills for patient-centered care. Health Affairs (Project Hope), 29, 1310-1318. 10.1377/hlthaff.2009.0450

Levinson, W., Roter, D. L., Mullooly, J. P., Dull, V. T., \& Frankel, R. M. (1997). Physician-patient communication: The relationship with malpractice claims among primary care physicians and surgeons. JAMA: Journal of the American Medical Association, 277, 553-559.

10.1001/jama.1997.03540310051034 
Makowski, S. K. E., \& Epstein, R. M. (2012). Turning toward dissonance: Lessons from art, music, and literature. Journal of Pain and Symptom Management, 43, 293-298. 10.1016/j.jpainsymman.2011.06.014

Matthews, D. A., Suchman, A. L., \& Branch, W. T., Jr. (1993). Making “connexions”: Enhancing the therapeutic potential of patient-clinician relationships. Annals of Internal Medicine, 118, 973-977. 10.7326/0003-4819-118-12-199306150-00010

Mauksch, L. B., Dugdale, D. C., Dodson, S., \& Epstein, R. (2008). Relationship, communication, and efficiency in the medical encounter: Creating a clinical model from a literature review. Archives of Internal Medicine, 168, 1387-1395. 10.1001/archinte.168.13.1387

McPhee, S. J. (1981, April17). The practice of presence. Paper presented at the Alpha Omega Alpha Honor Society Spring Lecture, Piscataway, NJ.

Mengel, M. B., \& Mauksch, L. B. (1989). Disarming the family ghost: A family of origin experience. Family Medicine, 21, 45-49.

Miller, W. L. (1992). Routine, ceremony, or drama: An exploratory field study of the primary care clinical encounter. The Journal of Family Practice, 34, 289-296.

Mishler, E. G., Clark, J. A., Ingelfinger, J., \& Simon, M. P. (1989). The language of attentive patient care: A comparison of two medical interviews. Journal of General Internal Medicine, 4, 325-335. 10.1007/BF02597407

Moore, J. (2008). What Sir Luke Fildes’ 1887 painting The Doctor can teach us about the practice of medicine today. The British Journal of General Practice, 58, 210-213. 10.3399/bjgp08X279571 
Peabody, F. W. (1927). The care of the patient. JAMA: Journal of the American Medical Association, 88, 877-882.

Rabow, M. W., \& McPhee, S. J. (1999). Beyond breaking bad news: How to help patients who suffer. The Western Journal of Medicine, 171, 260-263.

Rao, J. K., Anderson, L. A., Inui, T. S., \& Frankel, R. M. (2007). Communication interventions make a difference in conversations between physicians and patients: A systematic review of the evidence. Medical Care, 45, 340-349. 10.1097/01.mlr.0000254516.04961.d5

Saba, G. W., Wong, S. T., Schillinger, D., Fernandez, A., Somkin, C. P., Wilson, C. C., \& Grumbach, K. (2006). Shared decision making and the experience of partnership in primary care. Annals of Family Medicine, 4, 54-62. 10.1370/afm.393

Schouten, B. C., \& Meeuwesen, L. (2006). Cultural differences in medical communication: A review of the literature. Patient Education and Counseling, 64, 21-34. 10.1016/j.pec.2005.11.014

Starfield, B. (2011). Is patient-centered care the same as person-focused care?The Permanente Journal, 15, 63-69. 10.7812/TPP/10-148

Stewart, M., Brown, J. B., Donner, A., McWhinney, I. R., Oates, J., Weston, W. W., \& Jordan, J. (2000). The impact of patient-centered care on outcomes. The Journal of Family Practice, 49, 796-804. 
Street, R. L., Jr., Makoul, G., Arora, N. K., \& Epstein, R. M. (2009). How does communication heal? Pathways linking clinician-patient communication to health outcomes. Patient Education and Counseling, 74, 295-301. 10.1016/j.pec.2008.11.015

Suchman, A. L. (2006). A new theoretical foundation for relationship-centered care: Complex responsive processes of relating. Journal of General Internal Medicine, 21(Suppl. 1), S40-S44. 10.1111/j.15251497.2006.00308.x

Suchman, A. L., Markakis, K., Beckman, H. B., \& Frankel, R. (1997). A model of empathic communication in the medical interview. JAMA: Journal of the American Medical Association, 277, 678-682. 10.1001/jama.1997.03540320082047

Suchman, A. L., \& Matthews, D. A. (1988). What makes the patient-doctor relationship therapeutic? Exploring the connexional dimension of medical care. Annals of Internal Medicine, 108, 125-130. 10.7326/0003-4819-108-1-125

Tulsky, J. A. (2005). Beyond advance directives: Importance of communication skills at the end of life. JAMA: Journal of the American Medical Association, 294, 359-365. 10.1001/jama.294.3.359

Ventres, W. B. (2012a). How I think: Perspectives on process, people, politics, and presence. Journal of the American Board of Family Medicine, 25, 930-936. 10.3122/jabfm.2012.06.120093

Ventres, W. B. (2012b). The joy of family practice. Annals of Family Medicine, 10, 264-268. 10.1370/afm.1372 
Ventres, W. B., \& Frankel, R. M. (2010). Patient-centered care and electronic health records: It's still about the relationship. Family Medicine, 42, 364-366.

Ventres, W. B., \& Haq, C. (2014). Toward a cultural consciousness of self in relationship: From "us and them” to “we.”Family Medicine, 46, 691-695.

Verghese, A. (2009). A touch of sense. Health Affairs (Project Hope), 28, 1177-1182.

10.1377/hlthaff.28.4.1177

Verghese, A., Brady, E., Kapur, C. C., \& Horwitz, R. I. (2011). The bedside evaluation: Ritual and reason. Annals of Internal Medicine, 155, 550-553. 10.7326/0003-4819-155-8-201110180-00013

Weiss, B. D. (2014). How to bridge the health literacy gap. Family Practice Management, 21, 14-18.

Wilson, H. J. (2000). The myth of objectivity: Is medicine moving towards a social constructivist medical paradigm?Family Practice, 17, 203-209. 10.1093/fampra/17.2.203 
Table 1.

Clarifying Details for Figures 1 Through 5

To emphasize the essential aspects of shared presence, we:

- Center the words shared presence-Shared presence is the focal point for exploring the rich texture of communication between physicians, patients, and their families.

- Locate four sets of elemental factors around shared presence-The recurring interplay of these factors offers the best opportunity for shared presence and ultimately improved outcomes to occur.

- Identify shades, list numbers, and draw arrows-These shades, numbers, and arrows indicate how the distinct factors either shape or are shaped by shared presence.

- Use action verbs-Shared presence does not happen on its own but is an emergent and dynamic communicative process that requires foresight, practice, and engagement. 
Figure 1. Summary figure: shared presence and its four sets of elementary factors.

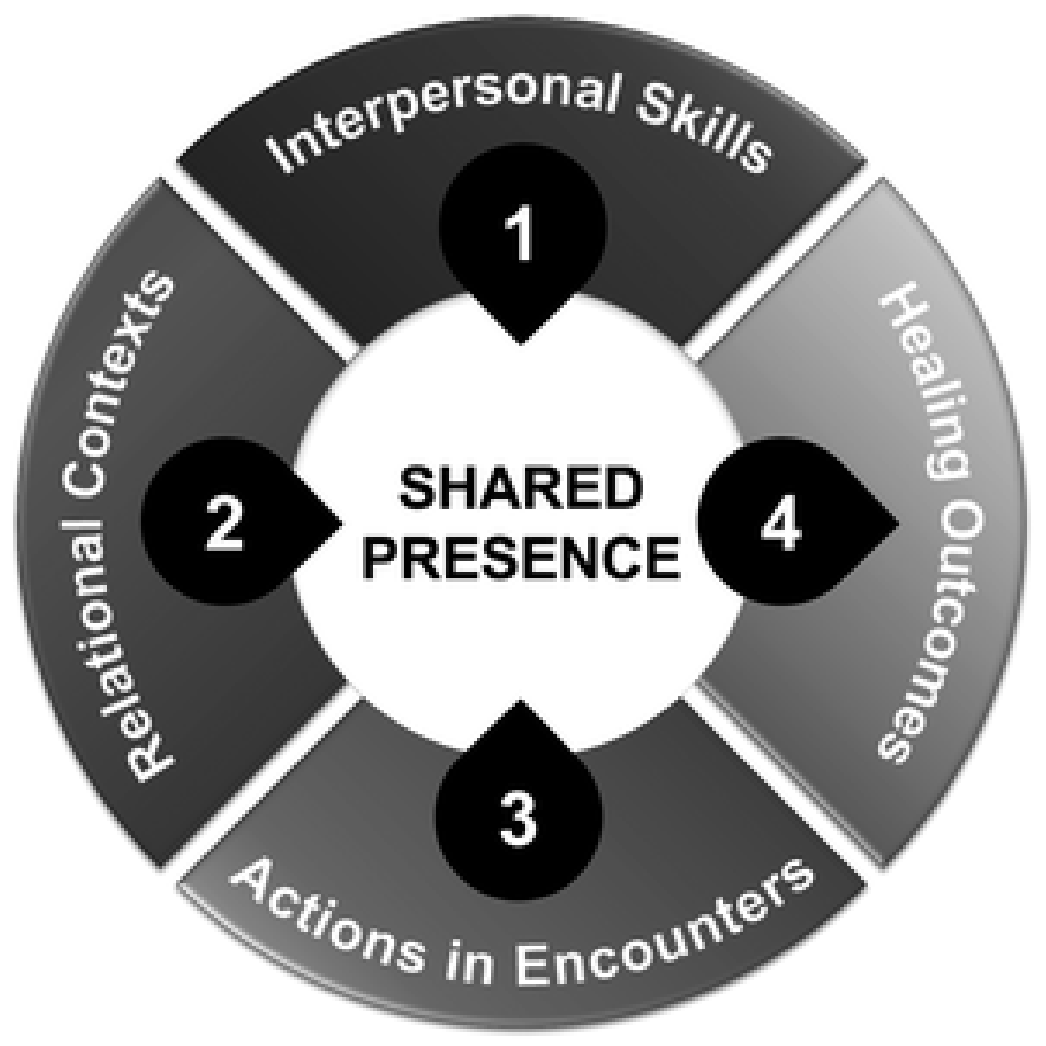


Figure 2. Interpersonal skills-exploring patient concerns and "seeing" the patient.




Figure 3. Relational contexts-understanding and integrating biopsychosociospiritual issues. IT/EHR = information technologies/electronic health records.

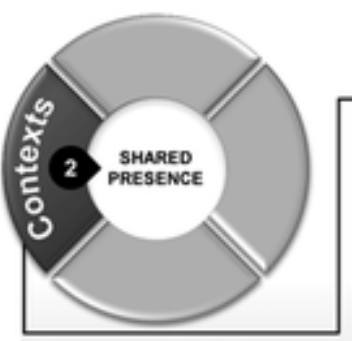

\section{Relational Contexts}

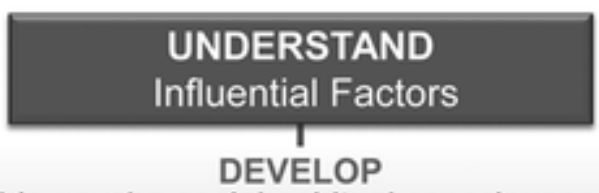

a bio-psycho-social-spiritual consciousness

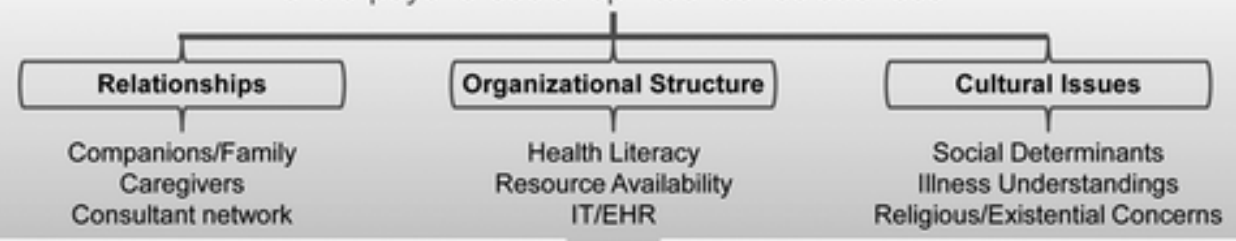

\section{CONSIDER}

Patient Strengths and Resiliencies

INTEGRATE

contextual issues into

clinical inquiry and plan development

SHARED PRESENCE 
Figure 4. Actions in the clinical encounter-developing habits of practice and addressing clinical issues.

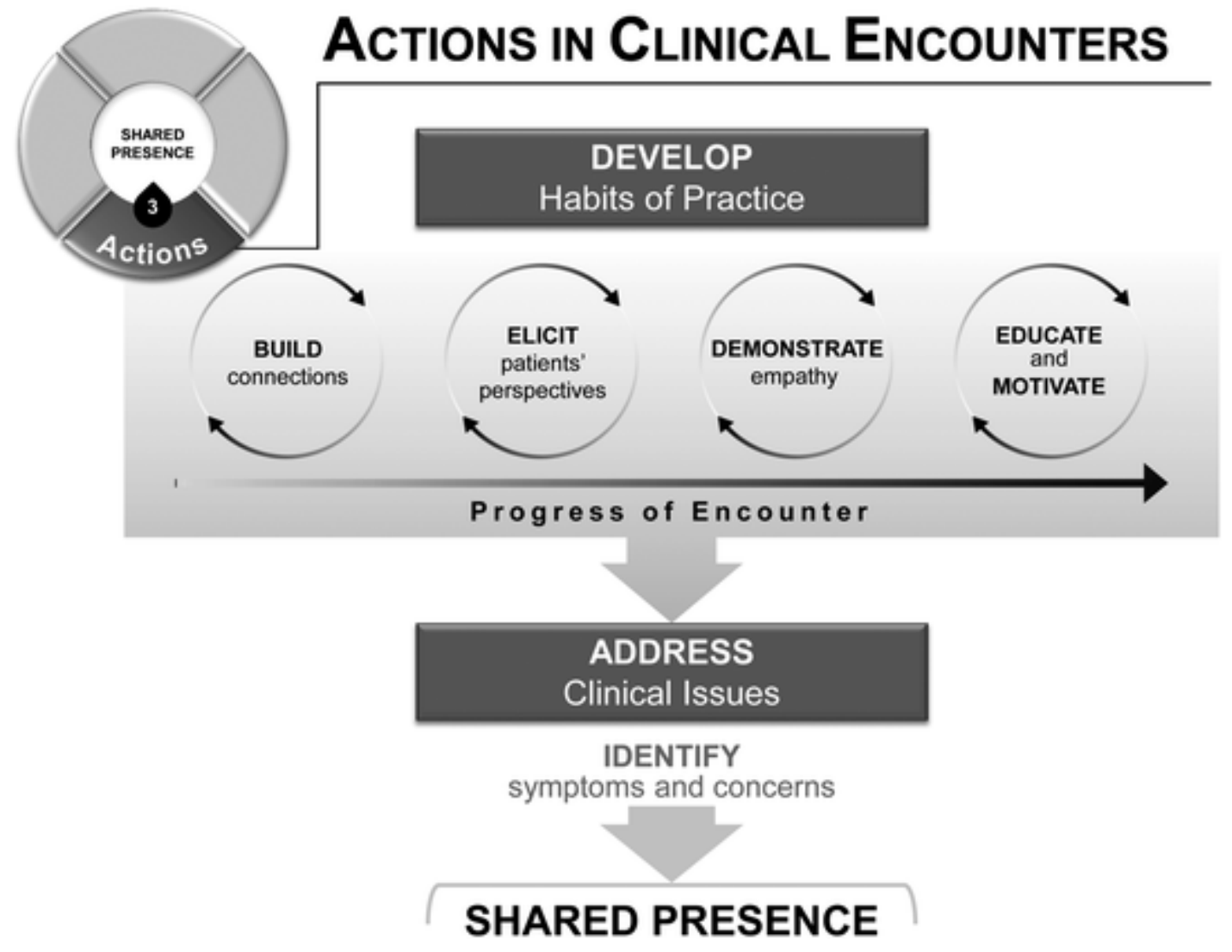


Figure 5. Healing outcomes-optimizing process and therapeutic outcomes.



